\title{
Effect of machining polarity on single discharge characteristics in micro-EDM
}

\author{
Kan Wang ${ }^{*}$, Yong Liu \\ School of Mechanical, Electrical \& Information Engineering, Shandong University, Weihai, Shandong, 264209, China
}

\begin{abstract}
In micro electrical discharge machining (micro-EDM), polarity effect is attributed to the difference in energy distribution into the anode and cathode. Understanding the effect of machining polarity on energy distribution bears significance in predicting and controlling machining performances. Single discharge experiments were conducted in this study, to explore single discharge characteristics. The plasma radius and energy distribution were calculated by combining the crater size and the electro-thermal model. The results show that the influence of discharge current on crater depth-to-diameter ratio (H/D) is not significant with positive polarity. The plasma radius, fraction of energy transferred to workpiece, and crater size are greater for micro-EDM with negative polarity than positive polarity.
\end{abstract}

\section{Introduction}

Electrical discharge machining (EDM) is an alternative approach to processing conductive materials, particularly for those that are difficult to cut. During EDM, the material is eroded because of intermittent spark discharges[1]. With rapid advances in microelectromechanical systems, micro-parts are more widely used in various applications, and the quality requirements imposed on them have become increasingly stringent. Compared with traditional processing technologies, micro-EDM presents evident advantages attributable to noncontact machining characteristics[2]. Material removal processes can be regarded as the accumulation of a large number of single discharge craters. Thus, numerous studies have been conducted on single discharge to explore the material removal mechanism. Wong et al.[3] found that the energy required to remove a unit volume of material at lower-energy discharges is considerably less than that at higher-energy discharges. The finite-volume method was used by Alwin Varghese et al.[4] to analyze temperature distribution by single discharge experiments.

Energy distribution is a key parameter for building a theoretical model of material erosion and determining the accuracy of the theoretical model[5,6]. The energy distribution is not constant and is influenced by the electrode material, dielectric, and machining parameters. Single discharge experiments can be used to study energy distribution during EDM. Zhang et al.[7]proposed a method to determine energy distribution by comparing the melted boundary of a single discharge crater and the isothermal curve of the thermal-physical model. A similar method was applied by Shen et al.[8]. Their studies indicated that energy distribution was significantly affected by power density.
Singh[9]measured the temperature at different locations during single-pulse discharge and combined the heat transfer equations to determine the energy distribution. The results show that the fraction of energy transferred to the workpiece varies with current and pulse duration from $6.1 \%$ to $26.82 \%$.

The aforementioned studies reported on energy distribution during EDM processing from different perspectives and substantially contributed to explore the machining mechanism. However, most published articles about energy distribution were conducted for conventional EDM and only few refer to micro-EDM. The difference in tool electrode size and discharge energy between conventional EDM and micro-EDM leads to variations in their machining mechanism. Consequently, the energy transferred to the anode and cathode is different, which significantly results in polarity effect $[10,11]$.

Accordingly, the energy distribution in micro-EDM needs to be explored to improve the application of micro-EDM. This study was aimed at identifying the single discharge characteristics of micro-EDM with different polarities. Several single discharge tests with different polarities were conducted. The crater depth-todiameter ratio $(H / D)$, plasma radius, and energy distribution were discussed.

\section{Electro-thermal model}

The lateral section of plasma is simplified to be circular. The distances in directions parallel and vertical to the electrode surface are expressed as $r$ and $z$. In single discharge, the governing partial differential equation that determines temperature distribution can be expressed as follows:

* Corresponding author: wangkanxw@foxmail.com 


$$
\frac{1}{\alpha} \frac{\partial T}{\partial t}=\frac{\partial^{2} T}{\partial r^{2}}+\frac{1}{r} \frac{\partial T}{\partial r}+\frac{\partial^{2} T}{\partial z^{2}}
$$

where $T$ is the temperature distribution of the electrode, and $\alpha$ is the thermal diffusivity of the electrode material, which is the thermal conductivity divided by density and specific heat capacity.

In micro-EDM, extremely high local temperature and temperature gradient is generated on the electrode surface, and the material that reaches vaporization temperature is vaporized. The impulse force is extremely small, which renders the melted material difficult to remove. That is, for conventional EDM, fusion is the predominant material removal method, whereas for micro-EDM, vaporization is the primary technique used $[10,12]$. Thus, the latent heat of fusion and vaporization must be considered in micro-EDM. The equivalent thermal diffusivity is proposed and expressed as follows:

$$
\alpha=\frac{K_{\mathrm{t}}}{\rho\left(C_{\mathrm{p}}+\left(L_{\mathrm{m}}+L_{\mathrm{v}}\right) /\left(T_{\mathrm{v}}-T_{0}\right)\right)}
$$

where $K_{\mathrm{t}}$ is the thermal conductivity of the electrode material, $\rho$ is the material density, $C_{\mathrm{p}}$ is the specific heat, $L_{\mathrm{m}}$ is the latent heat of fusion, $L_{\mathrm{v}}$ is the latent heat of vaporization, $T_{\mathrm{v}}$ is the vaporization temperature, and $T_{0}$ is the ambient temperature.

The initial temperature of the electrode is assumed to be equal to ambient temperature. A disk heat source is adopted in this model to simulate the heat transferred to the electrode during discharge.

The exact solution of Eq. (1) is given below.

$T(r, z, t)=T_{0}+\frac{F V l}{2 \pi K_{\mathrm{t}} R} \int_{0}^{\infty} J_{0}(\lambda r) J_{1}(\lambda R) \times$

$\left[e^{-\lambda z} \operatorname{erfc}\left(\frac{z}{2 \sqrt{\alpha t_{d}}}-\lambda \sqrt{\alpha t_{d}}\right)-e^{\lambda z} \operatorname{erfc}\left(\frac{z}{2 \sqrt{\alpha t_{d}}}+\right.\right.$

$\left.\left.\lambda \sqrt{\alpha t_{d}}\right)\right] \frac{d \lambda}{\lambda}$

where $F$ is the fraction of energy transferred to the anode or cathode, $V$ is the discharge voltage, $I$ is the discharge current, $R$ is the plasma radius on the workpiece surface, $J_{0}$ and $J_{1}$ are the zero-order and first-order Bessel functions of the first kind, respectively. The temperature distribution of the electrode material is given by Eq. (3).

\section{Determination of plasma radius and fraction of energy transferred to the electrode}

The most direct method of obtaining plasma radius is the use of a high-speed video camera. Kunieda et al. [13] recorded plasma expansion by using a high-speed video camera. Their study played a significant role in exploring energy transfer. However, their research method was not suitable for micro-EDM. The discharge duration of micro-EDM is microsecond-level, even submicrosecond-level generally. The highest frame rate of a high-speed video camera is thus far several million fps. Only few frames - even none-could be captured in single discharge process of micro-EDM. High-speed video photography cannot meet the requirements of micro-EDM.
As previously mentioned, vaporization is the predominant material removal manner for micro-EDM. The lower the discharge energy, the larger the fraction of material removed by vaporization[12]. The assumption that the material is entirely removed by vaporization in micro-EDM is proposed to calculate the plasma radius and fraction of energy transferred to the electrode. Enumeration is used to obtain an approximate solution. The solution process is presented in Fig. 1.

\section{Experiment setup and results}

Single discharge experiments were conducted with positive polarity (with the tool as the anode and the workpiece as the cathode) and negative polarity (with the tool as the cathode and the workpiece as the anode). The tool electrode and workpiece materials were brass and austenitic stainless steel (AISI 304) respectively. A digital storage oscilloscope was used to capture the discharge current and discharge voltage. The discharge duration is not constant owing to the breakdown delay time and randomness of discharge, which can be obtained from the captured discharge waveform. The experimental results are listed in Tables 1 and 2. The typical craters are shown in Fig. 2.

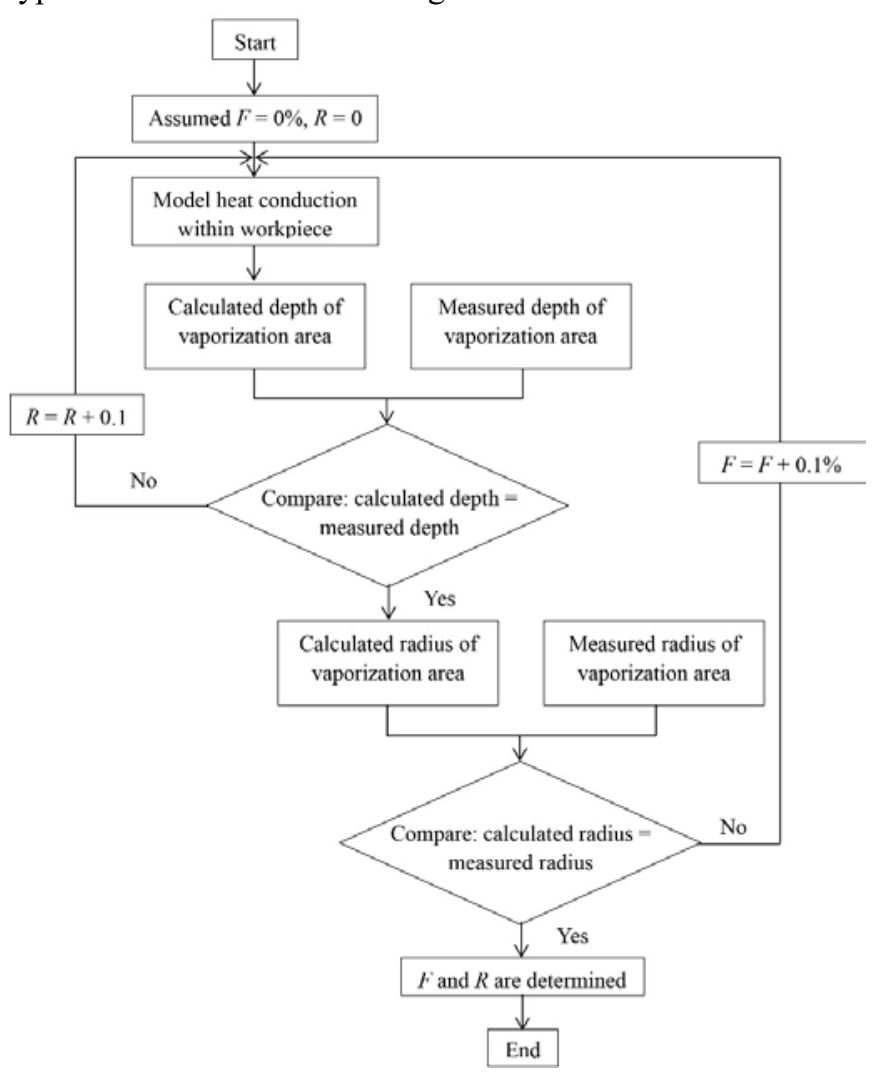

Fig.1. Flow chart of determining $F$ and $R$

The nonlinear regression models of the plasma radius for discharge current and discharge duration when machining with negative polarity (the workpiece as the anode) $\left(R_{\mathrm{a}}\right)$ and positive polarity (the workpiece as the cathode) $\left(R_{\mathrm{c}}\right)$ are given as follows:

$$
R_{\mathrm{a}}=7.685 I^{0.437} t^{0.3764}
$$




$$
R_{\mathrm{c}}=5.0189 I^{0.669} t^{0.3726}
$$

Fig. 3 shows the influence of discharge duration and discharge current on plasma radius. The plasma radius increases with an increase in discharge current and discharge duration. The increase in discharge current leads to increases in pressure and temperature gradients, which benefits plasma expansion. On the other hand, when a longer discharge duration is adopted, sufficient time is provided for plasma expansion. On the basis of the two scenarios, the plasma radius increases with an increase in discharge current and discharge duration.

Table 1. Experimental results of single discharge with negative

\begin{tabular}{cccccc}
$\begin{array}{c}\text { Dischar } \\
\text { ge } \\
\begin{array}{c}\text { current } \\
\text { (A) }\end{array}\end{array}$ & $\begin{array}{c}\text { Dischar } \\
\text { ge } \\
\text { duration } \\
\text { (us) }\end{array}$ & $\begin{array}{c}\text { Crater } \\
\text { diamet } \\
\text { er } \\
(\mu \mathrm{m})\end{array}$ & $\begin{array}{c}\text { Crater } \\
\text { depth } \\
(\mu \mathrm{m})\end{array}$ & $\begin{array}{c}\text { Plasma } \\
\text { radius } \\
(\mu \mathrm{m})\end{array}$ & $\begin{array}{c}\text { Fraction of } \\
\text { energy } \\
\text { transferred } \\
\text { to workpiece } \\
(\%)\end{array}$ \\
\hline 0.61 & 0.23 & 5.43 & 1.10 & 2.8 & 7.8 \\
0.41 & 0.50 & 7.33 & 1.34 & 3.9 & 15.4 \\
0.63 & 0.74 & 10.75 & 1.99 & 5.7 & 14.7 \\
0.74 & 0.61 & 11.34 & 1.94 & 6.2 & 13.2 \\
0.98 & 0.85 & 11.45 & 1.62 & 6.8 & 10.2 \\
1.07 & 0.99 & 14.82 & 2.23 & 8.6 & 12.0 \\
1.04 & 0.27 & 8.40 & 1.49 & 4.6 & 7.0 \\
1.13 & 0.75 & 12.04 & 1.76 & 7.1 & 9.3 \\
1.23 & 0.52 & 10.74 & 1.65 & 6.1 & 7.5 \\
1.44 & 0.67 & 13.45 & 2.03 & 7.8 & 8.1 \\
1.66 & 0.36 & 12.26 & 2.06 & 6.8 & 6.4 \\
1.59 & 0.96 & 14.51 & 1.82 & 9.3 & 8.2 \\
1.66 & 0.22 & 10.80 & 1.62 & 6.2 & 5.6 \\
1.98 & 0.97 & 16.03 & 2.31 & 9.6 & 7.1 \\
1.72 & 0.38 & 12.97 & 2.01 & 7.4 & 6.5 \\
\hline
\end{tabular}

Table 2. Experimental results of single discharge with positive

\begin{tabular}{cccccc}
$\begin{array}{c}\text { Dischar } \\
\text { ge } \\
\text { current } \\
(\mathrm{A})\end{array}$ & $\begin{array}{c}\text { Dischar } \\
\text { ge } \\
\text { duration } \\
\text { (us) }\end{array}$ & $\begin{array}{c}\text { Crater } \\
\text { diame } \\
\text { ter } \\
(\mu \mathrm{m})\end{array}$ & $\begin{array}{c}\text { Crater } \\
\text { depth } \\
(\mu \mathrm{m})\end{array}$ & $\begin{array}{c}\text { Plasma } \\
\text { radius } \\
(\mu \mathrm{m})\end{array}$ & $\begin{array}{c}\text { Fraction of } \\
\text { energy } \\
\text { transferred } \\
\text { to workpiece } \\
(\%)\end{array}$ \\
\hline 0.54 & 0.59 & 3.52 & 0.52 & 2.1 & 5.7 \\
0.48 & 0.15 & 1.76 & 0.41 & 0.9 & 3.3 \\
0.66 & 0.40 & 4.04 & 0.64 & 2.3 & 5.3 \\
0.57 & 0.79 & 7.00 & 1.11 & 4.1 & 10.8 \\
0.88 & 0.23 & 6.80 & 1.33 & 3.6 & 6.7 \\
0.82 & 0.65 & 7.92 & 1.18 & 4.6 & 8.4 \\
1.02 & 0.65 & 4.96 & 0.69 & 2.9 & 4.2 \\
0.99 & 0.48 & 4.04 & 0.68 & 2.2 & 3.5 \\
1.08 & 0.35 & 8.62 & 1.45 & 4.8 & 6.9 \\
1.22 & 0.73 & 9.16 & 1.15 & 6.3 & 6.7 \\
1.01 & 0.68 & 6.98 & 1.00 & 4.1 & 6.0 \\
1.17 & 0.26 & 5.20 & 0.97 & 2.7 & 3.8 \\
1.92 & 0.76 & 13.20 & 1.77 & 8.2 & 6.1 \\
1.98 & 0.89 & 9.26 & 1.16 & 5.9 & 4.2 \\
1.72 & 0.76 & 10.72 & 1.44 & 7.3 & 5.5 \\
\hline
\end{tabular}

The plasma radius is greater for negative polarity than positive polarity, which could be attributed to the difference in acceleration between a positive ion and an electron in plasma. Positive ion mass is significantly greater than electron mass. Compared with an electron colliding with the anode surface, a positive ion requires more time to collide with the cathode. In micro-EDM, the shortest possible discharge duration is generally accepted. For another, considerably more time is consumed for the ionization of a neutral particle, acceleration of a positive ion, and particle collisions. It indicates that only the positive ions near the cathode surface can collide with the workpiece surface. The plasma radius is greater on the anode surface than on the cathode surface.
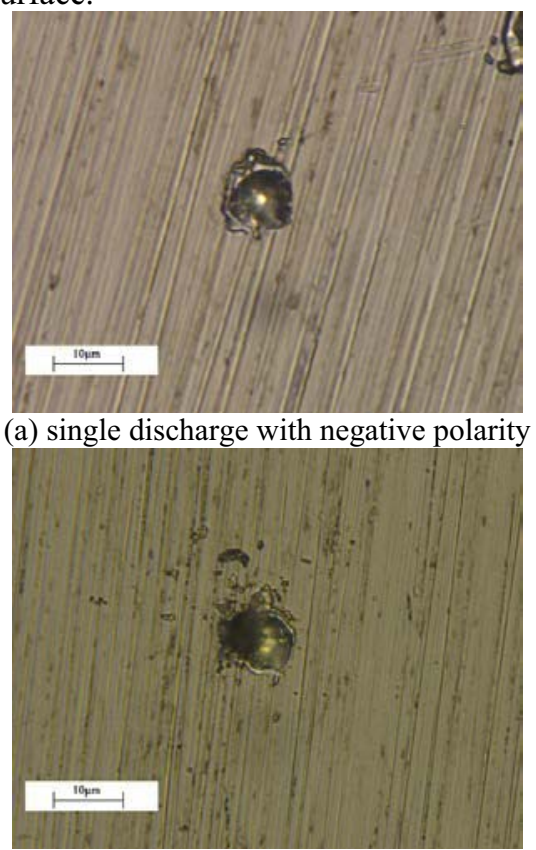

(b) single discharge with positive polarity

Fig. 2 Craters of single discharge

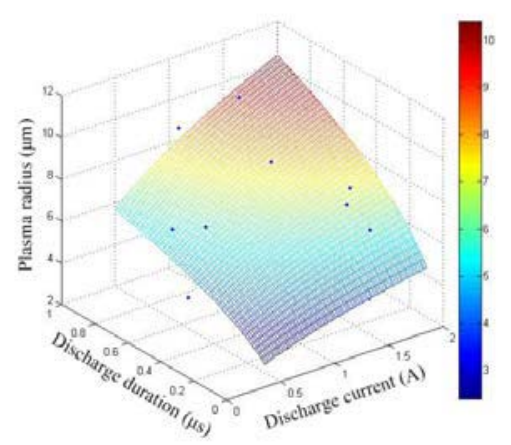

(a) machining with negative polarity

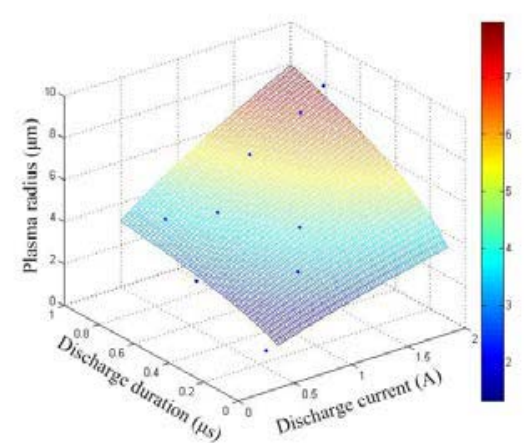

(b) machining with positive polarity

Fig.3 Surface plot of plasma radius versus discharge current and discharge duration 
The regression equations of $H / D$ for discharge current and discharge duration when machining with negative polarity $\left(H / D_{\mathrm{a}}\right)$ and positive polarity $\left(H / D_{\mathrm{c}}\right)$ are given as follows:

$$
\begin{aligned}
& H / D_{\mathrm{a}}=0.1499 I^{-0.1838} t^{-0.1241} \\
& H / D_{\mathrm{c}}=0.1273 I^{-0.089} t^{-0.2809}
\end{aligned}
$$

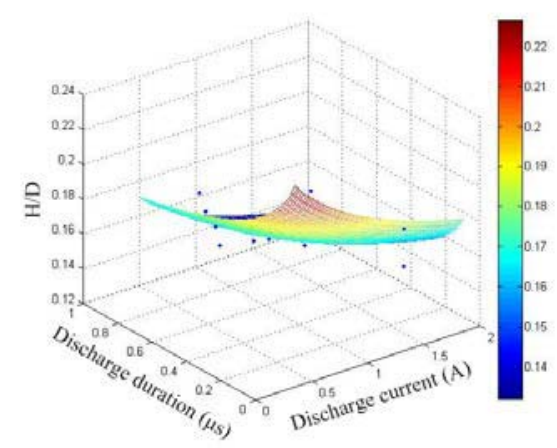

(a) machining with negative polarity

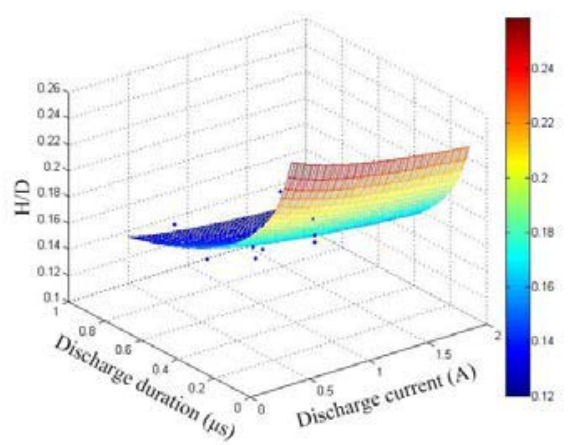

(b) machining with positive polarity

Fig.4 Surface plot of H/D versus discharge current and discharge duration

Fig. 4 shows that $H / D$ evidently decreases as discharge current and discharge duration increase regardless of the polarity selected. The reason is that higher discharge current and discharge duration result in a larger plasma radius, which promotes energy diffusion within the workpiece. As shown in Eq. (3), the radial velocity of energy diffusion is higher than the vertical velocity. Thus, a crater with a smaller $H / D$ is generated when discharge current and discharge duration are increased.

The influence of discharge duration on $H / D$ is more significant than that of discharge current for positive polarity. Plasma expands without enough time when the discharge duration is shorter. It results in a smaller bombarded area and crater diameter. Meanwhile, when the discharge duration is longer, positive ions can be accelerated for longer periods of time. This condition implies that more positive ions can collide with the workpiece surface, and a larger bombarded area is generated. Thus, discharge duration exerts a considerable influence on $H / D$ for micro-EDM with positive polarity.

The nonlinear regression models of the fraction of energy transferred to workpiece when machining with negative polarity $\left(F_{\mathrm{a}}\right)$ and positive polarity $\left(F_{\mathrm{c}}\right)$ are given as:

$$
F_{\mathrm{a}}=11.8945 I^{-0.6236} t^{0.4025}
$$

$$
F_{\mathrm{c}}=7.6038 I^{-0.3959} t^{0.4383}
$$

Fig. 5 shows the variation in the fraction of energy transferred to the workpiece with discharge current and discharge duration. The fraction of energy transferred to the workpiece increases as discharge current decreases or discharge duration increases. The trend is consistent regardless of the polarity adopted. The variation in energy distribution can be explained by plasma expansion. Meanwhile, most discharge energy is consumed for the formation and expansion of plasma at the early stage of discharge. With an increase in discharge duration, plasma tends to reach the steady stage, and more energy would be transferred to the anode or cathode. That is, the fraction of energy transferred to the workpiece increases with an increase in discharge duration. Meanwhile, the increase in discharge current prompts an increase in plasma radius, implying potentially greater plasma consumption. Thus, the fraction of energy transferred to the workpiece decreases with the increase in discharge current.

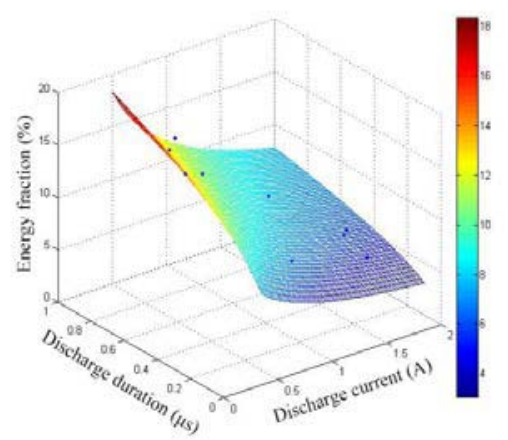

(a) machining with negative polarity

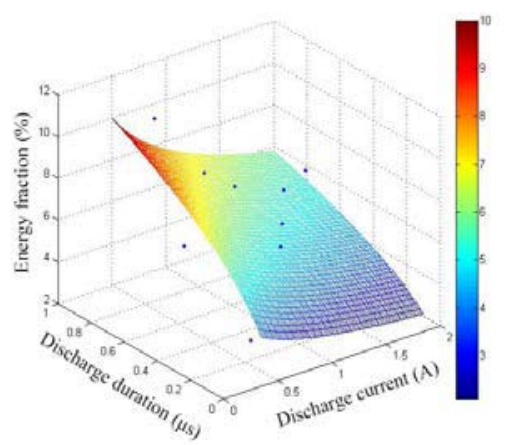

(b) machining with positive polarity

Fig. 5 Surface plot of the fraction of energy transferred to workpiece versus current and duration

In addition, Fig. 5 shows that the longer the discharge duration, the more significant the influence of discharge current on energy distribution. Plasma expansion is not significant when discharge duration is short. Thus, the fraction of energy transferred to the workpiece does not change significantly with the variation in discharge current and is often maintained at a lower level. Plasma can be further expanded when discharge duration is long. In this case, the decrease in discharge current leads to a decrease in plasma radius, implying that plasma can reach the steady stage early, which is favorable for the increase in the fraction of energy transferred to the workpiece. 


\section{Conclusions}

A single discharge experiment was conducted with both positive and negative polarity in this study. Main conclusions were drawn, as follows:

1. The plasma radius of negative polarity is greater than that of positive polarity. Plasma radius increases with increases in discharge current and discharge duration, and the trends of positive polarity and negative polarity are consistent.

2. The influence of discharge current on H/D is highly consistent with that of discharge duration for negative polarity. The influence of discharge duration on $\mathrm{H} / \mathrm{D}$ is more significant than that of discharge current for positive polarity.

3. The fraction of energy transferred to the workpiece increases as discharge current decreases or discharge duration increases regardless of the polarity adopted. The energy transferred to the workpiece and the crater size are greater for negative polarity than for positive polarity.

Authors acknowledge financial support from the China Postdoctoral Science Foundation (No. 2019M662347, 2018M630772), the National Key R\&D Program of China (No.2019YFB2001400), the Key R\&D Program of Shandong Province (No. 2019GGX104023), the Natural Science Foundation of Shandong Province (No. ZR2018MEE018).

\section{References}

1. Mascaraque-Ramírez C and Franco P 2020 Proceedings of the Institution of Mechanical Engineers, Part B: Journal of Engineering Manufacture. 234. 479-488.
2. D’Urso G, Giardini C and Ravasio C 2018 International Journal of Precision Engineering and Manufacturing.

3. Wong YS, Rahman M, Lim HS, Han H, Ravi N 2003 Journal of Materials Processing Technology, 140(13): 303-307.

4. Alwin Varghese, Kuriachen B, Satyananda Panda 2014 5th International \& 26th All India Manufacturing Technology, Design and Research Conference. 3271-3276.

5. DiBitonto DD, Eubank PT, Patel MR, e Barrufet MA 1989 Journal of Applied Physics; 66: 4095.

6. Yeo S, Kurnia W and Tan P 2007 Journal of Physics D: Applied Physics. 40: 2513.

7. Zhang Y, Liu Y, Shen Y, Li Z, Ji R, Cai B 2014 International Journal of Heat and Mass Transfer. 75: 425-432.

8. Shen Y, Liu Y, Zhang Y, Tan B, Ji R, Cai B 2014 International Journal of Advanced Manufacturing Technology; 70: 11-17.

9. Singh H 2012 International Journal of Heat and Mass Transfer. 55: 5053-5064.

10. Bor-Jenq W, Nannaji S and Ernest R 1992 Wear. 157: 31-49.

11. Zahiruddin M and Kunieda M 2010 Journal of Advanced Mechanical Design, Systems, and Manufacturing. 4: 1095-1106.

12. Zahiruddin M and Kunieda M 2012 CIRP AnnalsManufacturing Technology. 61: 187-190.

13. Kojima A, Natsu W and Kunieda M 2008 CIRP Annals - Manufacturing Technology. 57: 203-207. 